УДК 332.14 JEL R58

Гагарина Светлана Николаевна канд. экон. наук, ФГБОУ ВО «Калужский государственный университет им. К. Э. Циолковского», г. Калуга, Российская Федерация

ORCID: 0000-0003-4951-1832

e-mail: g_ug@mail.ru

Чаусов Николай Николаевич студент магистратуры, ФГБОУ ВО «Калужский государственный университет им. К. Э. Циолковского», г. Калуга, Российская Федерация

ORCID: 0000-0002-8210-983X

e-mail: straightman@list.ru

Левкина Вероника Николаевна студент, ФГБОУ ВО «Калужский государственный университет им. К. Э. Циолковского», г. Калуга, Российская Федерация

ORCID: 0000-0002-8904-8291

e-mail:veronia.levkina@mail.ru

\section{Gagarina Svetlana}

Candidate of Economic Sciences, Kaluga State University named after K. E. Tsiolkovski, Kaluga, Russia

ORCID: 0000-0003-4951-1832

e-mail: g_ug@mail.ru

\section{Chausov Nikolay}

Graduate Student, Kaluga State University named after K. E. Tsiolkovski, Kaluga, Russia

ORCID: 0000-0002-8210-983X

e-mail: straightman@list.ru

Levkina Veronika

Student, Kaluga State University named after K. E. Tsiolkovski, Kaluga, Russia

ORCID: 0000-0002-8904-8291

e-mail: veronia.levkina@mail.ru

\section{ПОВЫШЕНИЕ ЭФФЕКТИВНОСТИ ГОРОДСКОЙ ТРАНСПОРТНОЙ ИНФРАСТРУКТУРЫ НА ОСНОВЕ ЦИФРОВЫХ ТЕХНОЛОГИЙ}

\begin{abstract}
Аннотация. Обоснована необходимость повышения эффективности функциоониования транспортной инфраструктуры, являющейся важной подсистемой градообслуживающей сферы, как детерминанты качества жизни населения города. Выделены фактоpы, определяюшие качество функиионирования городской транспортной системы, особенности городских транспортных потоков. Проведен анализ развития транспортной инфраструктуры в России. Обосновано, что в условиях формирования ичифровой экономики эффективным инструментом принятия решений являются системы искусственного интеллекта. При формировании интеллектуальных систем управления городскими транспортными потоками предложено использование сетевых моделей, для которых математическими методами необходимо получать не только точечные, но и интервальные оценки параметров модели с учетом априорной неопределенности.
\end{abstract}

Ключевые слова: интервальные оценки, качество жизни, методы оптимизации, неопределенность, сетевые модели, транспортная инфраструктура, иифровые технологии, эффективность.

Цитирование: Гагарина С.Н., Чаусов Н.Н., Левкина В.Н. Повышение эффективности городской транспортной инфраструктуры на основе цифровых технологий//Вестник университета. 2020. № 7. C. $68-75$.

\section{IMPROVING THE EFFICIENCY OF URBAN TRANSPORT INFRASTRUCTURE BASED ON DIGITAL TECHNOLOGIES}

\begin{abstract}
The need to improve the efficiency of transport infrastructure, which is an important subsystem of urban services as a determinant of the quality of life of the city's population, has been substantiated. The factors that determine the quality of the urban transport system, the features of urban transport have been highlighted. Transport infrastructure development in Russia has been analysed. It has been proved that in the conditions of the formation of the digital economy, artificial intelligence systems are an effective tool for decision-making. In the formation of intelligent systems for managing urban transport flows, the use of network models has been proposed, for which mathematical methods are necessary to obtain not only point, but also interval estimates of the model parameters, taking into account a priori uncertainty.
\end{abstract}

Keywords: digital technologies, efficiency, interval estimates, life quality, network models, optimization methods, transport infrastructure, uncertainty.

For citation: Gagarina S.N., Chausov N.N., Levkina V.N. (2020) Improving the efficiency of urban transport infrastructure based on digital technologies. Vestnik universiteta. I. 7, pp. 68-75. DOI: $10.26425 / 1816-4277-2020-7-68-75$

Глобальным социально-экономическим процессом современности является урбанизация. В городах уже сейчас живет больше половины населения нашей планеты, а к 2050 г. удельный вес достигнет 70 \%. В Российской Федерации (далее - РФ) на 01 января 2020 г. удельный вес городского населения в общей численности населения составляет 74,7 \% (109548 440 чел.) [10].

Благодарности. Исследования проведены при финансовой поддержке Российского фонда фундаментальных исследований (проект № 18-010-00672A).

Acknowledgements. Research was carried out with the financial support of the Russian Foundation for basic research (project no.18-01000672A).

() Гагарина С.Н., Чаусов Н.Н., Левкина В.Н., 2020. Статья доступна по лицензии Creative Commons «Attribution» («Атрибуция») 4.0. всемирная (http://creativecommons.org/licenses/by/4.0/).

The Author(s), 2020. This is an open access article under the CC BY 4.0 license (http://creativecommons.org/licenses/by/4.0/).

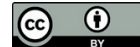


Одной из главных подсистем города, как сложной социально-экономической системы, является градообслуживающая сфера (городское хозяйство). Градообслуживающая сфера, или городское хозяйство, является первоосновой жизнеобеспечения муниципального образования. Главной целью функционирования градообслуживающей сферы является повышение качества жизни населения на соответствующей территории. Увеличение численности населения города и его территории предопределяют рост не только абсолютного размера предоставляемых организациями городского хозяйства услуг, но и удельных показателей (в расчете на одного жителя).

В 2017 г. впервые в истории России стартовал национальный проект «ЖКХ и городская среда». В рамках реализации этого проекта и государственной программы «Цифровая экономика» в 2018 г., в пилотном режиме стартовал ведомственный проект Министерства строительства и жилищно-коммунального хозяйства РФ «Умный город», который, начиная с 2019 г. стал обязательным. Проект «Умный город» направлен на повышение конкурентоспособности российских городов, формирование эффективной системы управления городским хозяйством, создание безопасных и комфортных условий для жизни горожан, на основе цифровых технологий и автоматизации процессов [12]. В марте 2019 г. Министерство строительства и ЖКХ России утвердило стандарт «Умный город». Реализация мероприятий стандарта будет осуществляться в основном в больших, крупных и крупнейших городах (с численностью населения свыше 100 тыс. человек), то есть охватит 15,3 \% общего количества городов России [13].

Важнейшим фактором развития на основе концепции умного города являются цифровые технологии [7]. В мировом масштабе использование цифровых технологий в экономической деятельности имеет высокий уровень дифференциации. При этом отмечаются опережающие темпы роста доли цифровой экономики в общем объеме валового внутреннего продукта (далее - ВВП) некоторых развивающихся стран, для которых цифровизация рассматривается как инструмент модернизации и повышения конкурентоспособности национальной экономики, по сравнению с развитыми странами, рассматривающими цифровые технологии как инструмент сохранения ведущих позиций в мировой экономике.

В 2018 г. удельный вес цифровой экономики в общем объеме ВВП России снизился на 0,2 п. п. по сравнению с уровнем 2016 г. и составил 2,6 \%, что ниже аналогичного показателя стран-лидеров в 2-3 раза [2]. Отставание от мирового уровня характерно и для российского транспортного комплекса. Однако в нем наблюдается положительная динамика по внедрению инновационных проектов на основе цифровых технологий.

Устойчивое развитие города предполагает стабильность его функционирования и является результатом эффективного управления составляющими элементами города как сложной социо-эколого-экономической системы [3]. Особую актуальность на современном этапе имеют вопросы управления объектами территориальной инфраструктуры, в частности транспортной системой. Это обусловлено повышением уровня автомобилизации (каждый третий россиянин является автовладельцем) и тем, что во многих городах, несмотря на стимулирование со стороны властей использования общественного транспорта, личные автомобили остаются основным средством передвижения, что обостряет проблему загруженности транспортной сети города, увеличения количества аварий и, как следствие, снижает качество жизни населения соответствующей территории.

Взаимосвязь комплексного развития транспорта с другими отраслями хозяйства и социальной сферы определяет требования к транспорту в отношении направлений, объемов и качества перевозок, а также возможные пути его развития. Транспорт как инфраструктурная отрасль обеспечивает базовые условия жизнедеятельности общества, развитие транспорта является фактором, стимулирующим территориальное социально-экономическое развитие.

Комплексный характер развития пассажирского транспорта обусловлен, с одной стороны, сложной структурой транспорта, объединяющего в единую систему отдельные виды транспорта, с другой стороны, его особой ролью как инфраструктурной отрасли, обеспечивающей условия для экономического роста и повышения качества жизни населения.

Российская Федерация - многорегиональное государство с высоким уровнем не только межрегиональной, но и внутрирегиональной дифференциации. Возможности адаптации субъектов РФ к цифровой трансформации рассмотрены в [6]. Региональная неравномерность в развитии дорожной сети является сдерживающим фактором процесса выравнивания уровней социально-экономического развития регионов России.

Плотность автомобильных дорог с твердым покрытием в России увеличилась в 2018 г., по сравнению с уровнем 2005 г., более чем в 2 раза [10]. Максимальные значения данного показателя наблюдаются в регионах 
центральной и южной части РФ (рис. 1). Средняя плотность автомобильных дорог с твердым покрытием в четырех федеральных округах (Центральный, Южный, Северо-Кавказский, Приволжский) составила 282,57 км на

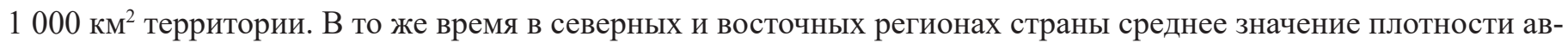
томобильных дорог составило 28,65 км дорог на 1000 км² территории.

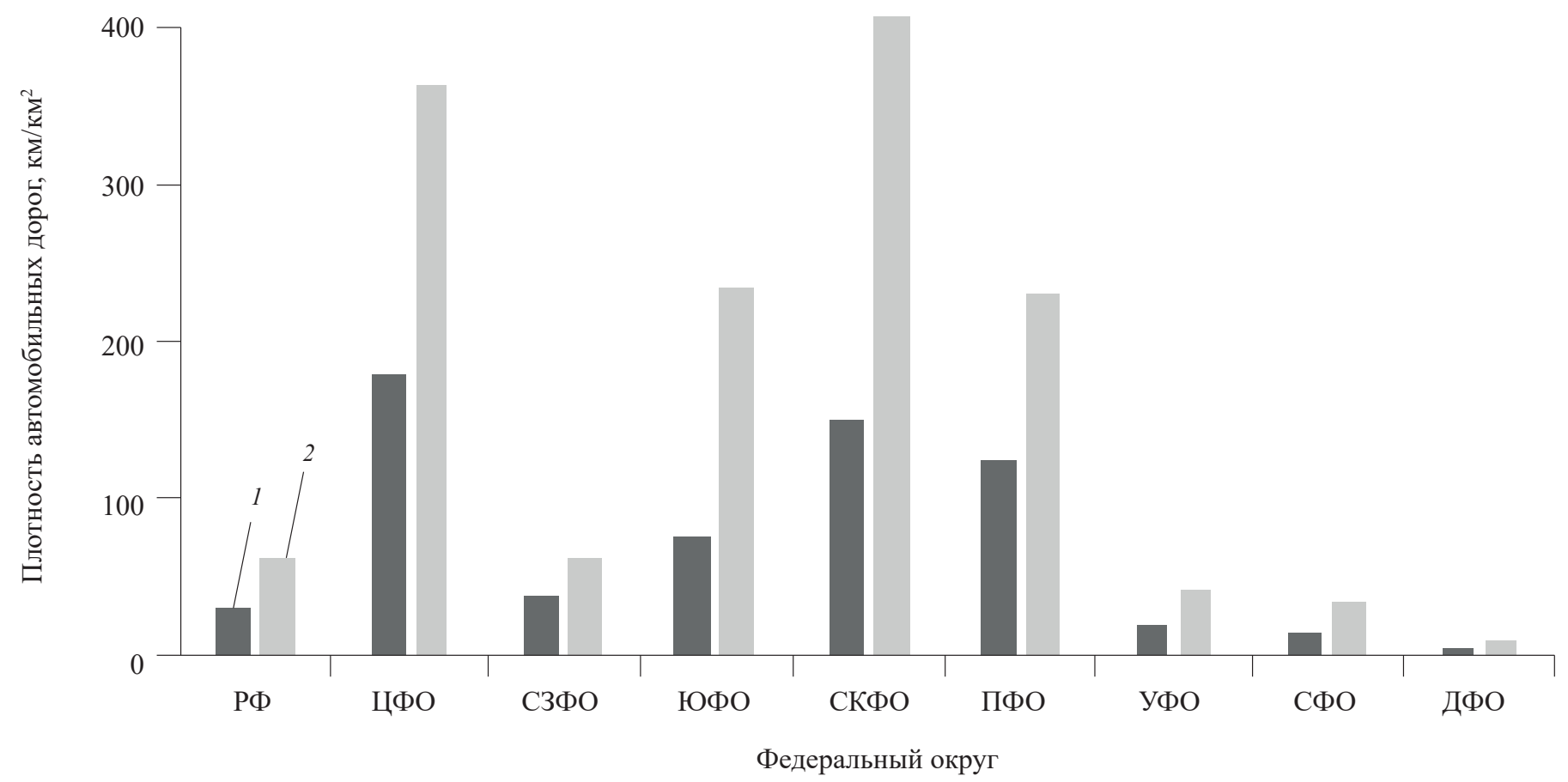

$1-2005$ г.; $2-2018$ г.

Составлено авторами по материалам источника [10]

Рис. 1. Плотность автомобильных дорог общего пользования с твердым покрытием в Российской Федерации и по федеральным округам (на конец 2005 г., 2018 г., км путей на 1000 км² территории)

В 2019 г., как и в 2018 г., Россия заняла 43 место из 141 страны в международном рейтинге конкурентоспособности Всемирного экономического форума (Global Competitiveness Index), получив 67 баллов из 100 возможных, что на 1,4 пункта превышает показатели 2018 г. Улучшив положение по некоторым позициям, Россия имеет низкую оценку по качеству автомобильных дорог (99-е место). При этом следует отметить позитивную динамику, в частности, в 2018 г. Россия занимала 114 позицию по данному показателю [9].

С 2008 г., после принятия Федерального закона от 8 ноября 2007 г. № 257-Ф3 «Об автомобильных дорогах и о дорожной деятельности в Российской Федерации и о внесении изменений в отдельные законодательные акты Российской Федерации», в регионах РФ активизировались работы по приведению автомобильных дорог в соответствие нормативным требованиям [1]. В результате проведенных мероприятий значительно увеличилась протяженность дорог местного значения с твердым покрытием. За 2008-2018 гг. сеть дорог местного значения увеличилась в 4,4 раза (с 124,1 тыс. км до 550,4 тыс. км) [10].

По данным официальной статистики доля автомобильных дорог общего пользования местного значения, отвечающих нормативным требованиям, на конец 2018 г., в среднем по РФ составляет 52,8 \% и характеризуется высоким уровнем вариативности. Размах вариации по федеральным округам составляет 34,8 \%. Максимальное значение данного показателя в Уральском федеральном округе - 73,6 \%, минимальное значение 38,8 \% - в Северо-Западном федеральном округе. По всем субъектам РФ вариативность составляет $96,2 \%$, что в 2,76 раза превышает размах вариации в разрезе федеральных округов. Среди субъектов, входящих в состав Центрального федерального округа, наибольшая доля автомобильных дорог общего пользования местного значения, отвечающих нормативным требованиям, в Белгородской области 80,3 \%, наименьшее значение данного показателя наблюдается в Ярославской области - 22,8 \% (размах вариации - 57,5 \%). 
В Калужской области доля автомобильных дорог общего пользования местного значения, отвечающих нормативным требованиям, ниже среднероссийского уровня и среднего значения по Центральному федеральному округу на 12,5 \% и 7,4 \% соответственно, и составила на конец 2018 г. 46,2 \% [10].

Среднероссийский уровень доли автомобильных дорог общего пользования регионального значения, отвечающих нормативным требованиям, ниже аналогичного показателя автомобильных дорог общего пользования местного значения (на конец 2018 г. 42,4 \%) [10]. В рамках реализации национального проекта «Безопасные и качественные дороги» к концу 2019 г. и 2024 г. нормативным требованиям должно соответствовать 44,1 \% и 50,9 \% автомобильных дорог регионального значения 83 субъектов РФ (в проекте не участвуют два субъекта - Республика Крым и г. Севастополь), что в абсолютном выражении составляет соответственно 221 и 255 тыс. км [11].

Важным требованием, предъявляемым к современным городским транспортным системам, является физическая безопасность. Уровень физической безопасности оценивается таким показателем, как «количество дорожно-транспортных происшествий в расчете на 100 тыс. человек населения», который в среднем по России имеет положительную динамику [3]. В 2018 г. «число дорожно-транспортных происшествий в расчете на 100 тыс. человек населения» уменьшилось по сравнению с уровнем 2005 г. на 26,4 \% [10]. Вариативность уровня физической безопасности транспортной инфраструктуры по федеральным округам представлена на рисунке 2.

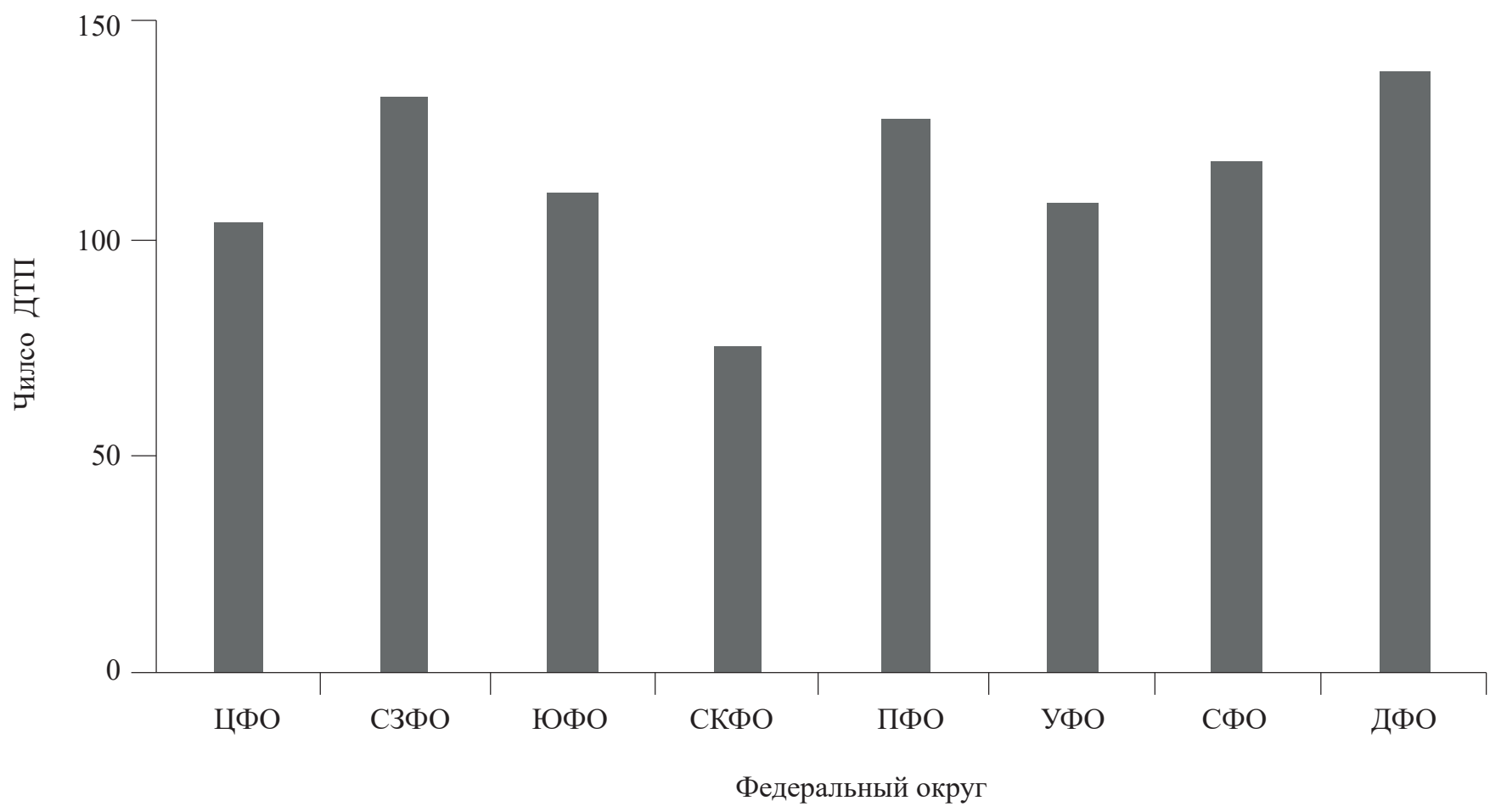

Составлено авторами по материалам источника [10]

Рис. 2. Число дорожно-транспортных происшествий в расчете на 100 тыс. человек населения по федеральным округам в 2018 г.

Проблема управления городскими транспортными потоками предполагает исследование их особенностей:

- случайный характер транспортных потоков допускает прогноз их параметров с определенной вероятностью;

- нестационарность транспортных потоков, параметры которых подвержены суточным, недельным и сезонным колебаниям;

- ограниченная управляемость, которая заключается в том, что управление транспортными потоками носит рекомендательный характер и при множестве влияющих факторов не дает возможность получить оптимальное решение;

- большое количество взаимосвязанных факторов, влияющих на поиск оптимального решения;

- отсутствие инструментальных средств для определения основных характеристик, определяющих качество управления транспортными потоками. 
При организации дорожного движения и проектировании оптимальной транспортной сети необходимо учитывать основные особенности и различные динамические характеристики транспортного потока, а также возможное влияние на эти характеристики внешних и внутренних факторов.

Наиболее перспективным вариантом решения данной проблемы в условиях цифровизации экономики является создание интеллектуальной системы управления городскими транспортными потоками, которая осуществляет автоматизированный сбор, обработку, анализ информации и выбор оптимальных управленческих решений.

Анализ зарубежного опыта свидетельствует о том, что интеллектуальные транспортные системы США, Японии, Германии, Франции и Китая рассматриваются в качестве интенсивного фактора решения транспортных проблем, механизма обеспечения национальной безопасности и нацелены на повышение эффективности поездок на городских магистралях, безопасности дорожного движения.

В качестве основы в процессе разработки искусственной интеллектуальной системы, в целях хорошего совпадения поведения искусственно созданной и естественной интеллектуальной системы, авторами предлагается исследование особенностей функционирования городских транспортных систем с применением сетевых моделей. Это объясняется тем, что современные транспортные, информационные, энергетические и другие территориально распределенные системы соответствуют сетевой структуре, для описания которой используется взвешенный граф. Ребрам и вершинам графа задают «веса», соответствующие характеристикам распределенных систем. Для нахождения оптимального решения в задачах на взвешенных графах оценивают экстремум функционала, заданного при определенных значениях весов вершин и ребер графа. Подобные задачи относятся к задачам линейного программирования. Широко используемыми для различных объектов территориальной инфраструктуры и эффективными методами нахождения оптимальных решений сетевых моделей являются методы линейного программирования.

При формировании модели необходимо определить условия существования оптимального решения в задачах линейного программирования [8]. При этом условия не являются неизменными, и особую значимость имеет анализ устойчивости принимаемого решения. Параметры сформированной модели включают коэффициенты целевой функции и матрицу, составленную из коэффициентов при неизвестных условиях-ограничениях. Изменение значений этих параметров приводит к изменению решения, и важно знать, при каких изменениях параметров оптимальное решение останется неизменным. Параметры модели могут меняться в результате изменения условий функционирования объектов территориальной инфраструктуры: меняется количество транспорта при перемещении из одного пункта в другой или изменяется пропускная способность сетевой модели. Все это приводит к неопределенности параметров модели. Предполагая, что параметры моделей задач линейного программирования являются случайными величинами можно оценить математическое ожидание и дисперсию этих случайных величин и для учета неопределенности параметров модели определить соответствующую им доверительную вероятность. В таких случаях, получение оптимального решения соответствует серии прямых близких задач с изменяемыми значениями параметров.

Учет неопределенности исходных данных может и не повлиять на оптимальное решение задачи линейного программирования. Однако наиболее часто небольшое изменение параметров модели приводит к изменению координат оптимальной точки решения. Кроме этого, неисследованным является интервальное оценивание параметров в задачах оптимизации сетевых моделей с учетом неопределенности исходной информации.

Рассмотрим математическую модель задачи линейного программирования в канонической форме:

$$
\boldsymbol{z}=\boldsymbol{c}^{\mathrm{T}}, \boldsymbol{x} \rightarrow \max , \text { при условиях } \boldsymbol{A x}=\boldsymbol{b}, x \geq 0,
$$

где $\boldsymbol{x}$ - вектор, определяющий исходные переменные; $\boldsymbol{A}$ - матрица размерности $m \times n$, включающая столбцы дополнительных переменных, преобразующих неравенства в равенства; $\boldsymbol{b}$ - вектор правых частей условий; $\boldsymbol{c}$ - вектор коэффициентов целевой функции.

В алгоритмах оптимизации транспортных сетей неопределенность исходной информации может быть отражена:

1) в неопределенности или погрешности коэффициентов $c$ целевой функции;

2) в неопределенности или погрешности элементов вектора $\boldsymbol{b}$ правой части условий-ограничений;

3) в неопределенности или погрешности элементов матрицы $\boldsymbol{A}$ системы ограничений. 
Задача о максимальном потоке позволяет оценить величину потока в системах, обладающих сетевой структурой. Каждой дуге сетевой структуры соответствует определенная пропускная способность. Введем обозначения: $s$ - узел - источник; $t$ - узел - сток; $v$ - поток, который входит в сеть в узле $s$ и выходит из сети в узле $t$. Запишем задачу о максимальном потоке в терминах задачи линейного программирования: найти максимум потока $v$ при ограничениях:

$$
\left.\begin{array}{l}
\sum_{k \in M_{O_{i}}} f_{k}-\sum_{k \in M_{T_{i}}} f_{k}=0, \quad i \in N-(s, t), \\
\sum_{k \in M_{O i}} f_{k}-\sum_{k \in M_{T i}} f_{k}-v=0, \\
\sum_{k \in M_{O i}} f_{k}-\sum_{k \in M_{T i}} f_{k}+v=0, \\
0 \leq v \leq v_{r}, \quad 0 \leq f_{k} \leq c_{k}, \quad k \in M,
\end{array}\right],
$$

где $N$ - набор узлов; $M$ - множество дуг; $O_{i}$ и $T_{i}-$ набор начальных и конечных узлов; $f_{k}-$ величина потока, проходящего по дуге $k ; c_{k}$ - максимальная (верхняя граница) пропускная способность дуги $k$.

В задаче о максимальном потоке величина потока, как правило, остается неизменной и сохраняется при перемещении по дугам сети, то есть $f_{k}^{\prime}=f_{k}$, где $f_{k}$ - величина потока в начале дуги $k, f_{k}^{\prime}-$ величина потока в конце дуги. В сетевых структурах значения потоков $f_{k}$ и $f_{k}^{\prime}$, а также пропускная способность $c_{k}$ дуги не являются постоянными и могут меняться в некоторых пределах. Например, поток транспортных систем зависит от времени суток, и в часы пиковой нагрузки его значение возрастает. Пропускная способность транспортной сети в часы пиковой нагрузки, наоборот, уменьшается.

Эти две величины не определяются однозначно, а имеют некоторую погрешность: $f_{k} \pm \Delta f_{k}$ и $c_{k} \pm \Delta c_{k}$. Учет погрешности $\Delta f_{k}$ приводит к тому, что значение потока по дуге сети может уменьшиться до нуля или стать отрицательным. Последний случай означает, что поток поменял свое направление. Учет погрешности $\Delta c_{k}$ может привести к уменьшению до нуля пропускной способности дуги.

Таким образом, неопределенность исходных данных может привести к изменению условий - ограничений, и соответственно изменить допустимые решения задачи линейного программирования, что в свою очередь отразится на оптимальном решении задачи.

Существует ряд статистических подходов, основанных на моделях «измерения с ошибками», позволяющих учесть погрешности $\Delta f_{k}$ и $\Delta c_{k}[4]$.

Например, существуют методы, в которых предлагается учитывать случайный характер аргументов функциональных зависимостей, а оценки параметров определяются методом наименьших квадратов, что приводит к несостоятельной оценке параметров. В некоторых подходах используют итеративный метод наименьших квадратов, в которых предполагается, что значения аргументов являются выборкой из некоторой генеральной совокупности с известной плотностью распределения и для построения минимизируемого функционала предлагается использовать разложение функциональной зависимости в ряд Тейлора в окрестности наблюдаемого значения аргумента. Анализ методов «измерений с ошибками» показывает простоту их применения для линейных функциональных зависимостей, а для оценивания параметров нелинейных функциональных зависимостей применяют сложные итерационные процедуры.

Во всех известных подходах учета погрешностей исходных данных является неисследованным интервальное оценивание параметров и функциональных зависимостей, оценки которых в ряде методов считаются точными. Кроме этого, как правило, не рассматриваются ситуации, когда параметры нескольких функций имеют близкие значения, соизмеримые с погрешностями измерений аргументов.

Для учета погрешностей исходных данных при оценивании параметров функциональных зависимостей используют методы конфлюэнтного анализа. В [5] доказано, что используемые в этих методах итерационные процедуры оценивания параметров являются сходящимися, а оценки параметров - несмещенными.

Таким образом, одним из ключевых ограничений экономического роста, качества жизни населения является низкая транспортная доступность. Обеспечить выполнение требований, предъявляемых со стороны 
населения, органов публичной власти и бизнеса возможно на основе технологической модернизации транспортной инфраструктуры. Модернизация и развитие транспортной системы является ориентиром для региональных и муниципальных властей при формировании и реализации социально-экономической политики. Это обусловлено тем, что эффективное функционирование транспортной системы способствует устойчивому и безопасному развитию территорий.

Несомненно, развитие транспортной инфраструктуры должно отвечать современным технологическим, социальным, экологическим вызовам. В связи с этим значительную роль в обеспечении эффективного функционирования и развития территориальных транспортных систем, повышения качества транспортных услуг играют цифровые технологии.

При разработке интеллектуальных систем управления городскими транспортными потоками целесообразным является использование сетевых моделей, для которых математическими методами необходимо получать не только точечные, но и интервальные оценки параметров модели с учетом априорной неопределенности, что способствует принятию оптимального решения и повышению эффективности функционирования городской транспортной инфраструктуры.

\section{Библиографический список}

1. Федеральный закон «Об автомобильных дорогах и о дорожной деятельности в Российской Федерации и о внесении изменений в отдельные законодательные акты Российской Федерации» от 8 ноября 2007 г. № 257-Ф3 (ред. от 01.03.2020) // СПС «КонсультантПлюс» [Электронный ресурс]. - Режим доступа: http://www.consultant.ru/document/cons_doc_ LAW_72386/ (дата обращения: 05.05.2020).

2. Абдрахманова, Г. И., Вишневский, К. О., Гохберг Л. М. [и др.]. Индикаторы цифровой экономики: 2019: статистический сборник; Нац. исслед. ун-т «Высшая школа экономики». - М.: НИУ ВШЭ, 2019. - 248 с.

3. Гагарина, С. Н., Чаусов, Н. Ю. Экономико-статистическая оценка как инструмент управления качеством жизни населения в целях устойчивого развития региона // Вестник университета. - 2016. - № 12. - С. 5-11.

4. Гагарин, Ю. Е., Гагарина, С. Н. Интервальное оценивание объемов потребления ресурсов при стохастических исходных данных // Вестник университета. - М.: ГУУ, 2018. - № 12. - С. 64-70. DOI: 10.26425/1816-4277-2018-12-64-70.

5. Гагарин, Ю. Е. Учет множества случайных факторов при использовании минимаксного критерия в задачах распознавания объектов // Моделирование, оптимизация и информационные технологии. - 2019. - Т. 7, № 1 (24). - С. 89-98.

6. Земцов, С., Баринова, В., Семенова, Р. Риски цифровизации и адаптация региональных рынков труда в России // Форсайт. - 2019. - Т. 13, № 2. - С. 84-96. DOI: 10.17323/2500-2597.2019.2.84.96.

7. Попов, Е. В., Семячков, К. А. Систематизация подходов к оценке развития умных городов // Экономика региона. $-2020 .-$ Т. 16, Вып. 1. - С. 14-27.

8. Таха, Х. А. Введение в исследование операций; 7-е изд.: пер. с англ. - М.: Издательский дом «Вильямс», 2007. -912 с.

9. Индекс глобальной конкурентоспособности [Электронный ресурс]. - Режим доступа: https:/gtmarket.ru/ratings/globalcompetitiveness-index/info (дата обращения: 30.04.2020).

10. Официальный сайт Федеральной службы государственной статистики [Электронный ресурс]. - Режим доступа: https:// gks.ru/folder/210/document/13204 (дата обращения: 01.05.2020).

11. Паспорт приоритетного проекта «Безопасные и качественные дороги» в редакции протокола от 21 ноября 2016 г. № 10 [Электронный ресурс]. - Режим доступа: http://static.government.ru/media/files/5EgAG7vBB8Y9iKqkhPBaGzh7dAml5H hz.pdf (дата обращения: 01.05.2020).

12. Проект «Умный город» [Электронный ресурс]. - Режим доступа: https://www.minstroyrf.ru/trades/gorodskaya-sreda/proekttsifrovizatsii-gorodskogo-khozyaystva-umnyy-gorod/ (дата обращения: 30.01.2020).

13. Стандарт «Умный город» [Электронный ресурс]. - Режим доступа: https:/www.minstroyrf.ru/upload/iblock/74f/Standart. $\operatorname{pdf}$ (дата обращения: 30.04.2020).

\section{References}

1. Federal'nyi zakon “Ob avtomobil'nykh dorogakh i o dorozhnoi deyatel'nosti v Rossiiskoi Federatsii i o vnesenii izmenenii v otdel'nye zakonodatel'nye akty Rossiiskoi Federatsii”' ot 8 noyabrya 2007 g. № 257-FZ (red. ot 01.03.2020) [Federal law "On Roads and Road Activities in the Russian Federation and on Amending Certain Legislative Acts of the Russian Federation" 
No. 257-FZ, dated on November 8, 2007]. Legal reference system “ConsultantPlus”. Available at: http://www.consultant.ru/ document/cons_doc_LAW_72386/(accessed 05.05.2020).

2. Abdrakhmanova G. I., Vishnevskii K. O., Gokhberg L. M. [et al]. Indikatory tsifrovoi ekonomiki: 2019: statisticheskii sbornik [Indicators of the digital economy: 2019: statistical compilation]. Nats. Issled. un-t "Vysshaya shkola ekonomiki" [National Research University "Higher School of Economics”]. Moscow, NIU VShE, 2019. 248 p.

3. Gagarina S. N., Chausov N. Yu. Ekonomiko-statisticheskaya otsenka kak instrument upravleniya kachestvom zhizni naseleniya $\mathrm{v}$ tselyakh ustoichivogo razvitiya regiona [Economic and statistical assessment as a tool for managing the quality of life of the population for the sustainable development of the region]. Vestnik universiteta, 2016, no. 12, pp. 5-11.

4. Gagarin Yu. E., Gagarina S. N. Interval'noe otsenivanie ob"emov potrebleniya resursov pri stokhasticheskikh iskhodnykh dannykh [Interval estimation of resource consumption with stochastic input data]. Vestnik universiteta, 2018, no. 12. pp. 64-70.

5. Gagarin Yu. E. Uchet mnozhestva sluchainykh faktorov pri ispol'zovanii minimaksnogo kriteriya v zadachakh raspoznavaniya ob"ektov [Taking into account many random factors when using the minimax criterion in object recognition problems]. Modelirovanie, optimizatsiya i informatsionnye tekhnologii [Modeling, Optimization and Information Technology], 2019, vol. 7, no. 1 (24), pp. 89-98.

6. Zemtsov S., Barinova V., Semenova R. Riski tsifrovizatsii i adaptatsiya regional'nykh rynkov truda v Rossii [Risks of digitalization and adaptation of regional labor markets in Russia]. Forsait [Foresight], 2019, vol. 13, no. 2, pp. 84-96.

7. Popov E. V., Semyachkov K. A. Sistematizatsiya podkhodov k otsenke razvitiya umnykh gorodov [Systematization of approaches to assessing the development of smart cities]. Ekonomika regiona [Economy of Region], 2020, vol. 16, no. 1, pp. 14-27.

8. Taha H. A. Vvedenie v issledovanie operatsii [Introduction to operations research]. Moscow, Izdatel'skii dom "Vil'yams", 2007. 912 p.

9. Indeks global'noi konkurentosposobnosti [Global Competitiveness Index]. Available at: https://gtmarket.ru/ratings/global-competitiveness-index/info (accessed 30.04.2020).

10. Ofitsial'nyi sait Federal'noi sluzhby gosudarstvennoi statistiki [Official website of the Federal State Statistics Service]. Available at: https://gks.ru/folder/210/document/13204 (accessed 01.05.2020).

11. Pasport prioritetnogo proekta “Bezopasnye i kachestvennye dorogi” v redaktsii protokola ot 21 noyabrya 2016 g. № 10 [Passport of the Priority Project "Safe and High-Quality Roads" in the version of the Protocol No. 10, dated on November 21, 2016]. Available at: http://static.government.ru/media/files/5EgAG7vBB8Y9iKqkhPBaGzh7dAml5Hhz.pdf (accessed 01.05.2020).

12. Proekt "Umnyi gorod" [Smart City Project]. Available at: https://www.minstroyrf.ru/trades/gorodskaya-sreda/proekt-tsifrovizatsii-gorodskogo-khozyaystva-umnyy-gorod/ (accessed 30.04.2020).

13. Standart "Umnyi gorod" [Smart City Standard]. Available at: https://www.minstroyrf.ru/upload/iblock/74f/Standart.pdf (accessed 30.04.2020). 